\title{
A New Kid on the Block. Le National Museum of the American Indian
}

Marie Mauzé et Joëlle Rostkowski

\section{(2) OpenEdition}

\section{Journals}

Édition électronique

URL : https://journals.openedition.org/jsa/1465

DOI : 10.4000/jsa.1465

ISSN : 1957-7842

\section{Éditeur}

Société des américanistes

\section{Édition imprimée}

Date de publication : 5 juin 2004

Pagination : 115-128

ISSN : 0037-9174

\section{Référence électronique}

Marie Mauzé et Joëlle Rostkowski, « A New Kid on the Block. Le National Museum of the American Indian », Journal de la Société des américanistes [En ligne], 90-2 | 2004, mis en ligne le 10 septembre 2014, consulté le 02 septembre 2022. URL : http://journals.openedition.org/jsa/1465 ; DOl : https:// doi.org/10.4000/jsa. 1465 


\title{
CHRONIQUE DU GROUPE D'INFORMATION \\ SUR LES AMÉRINDIENS
}

\author{
I. - ÉTATS-UNIS
}

\author{
A NEW KID ON THE BLOCK ${ }^{1}$ \\ LE NATIONAL MUSEUM OF THE AMERICAN INDIAN
}

\begin{abstract}
Ce musée marque un tournant dans l'histoire américaine, car les États-Unis commencent à prendre en considération l'histoire des premiers habitants du continent américain. ${ }^{2}$
\end{abstract}

Situé en plein cœur de Washington, en face du Capitole et à côté du musée de l'Air et de l'Espace, le National Museum of the American Indian (NMAI) est le petit dernier des dix-huit musées de la Smithsonian Institution. C'est en 1989 que le Congrès a voté la loi au titre de laquelle fut créé le musée. Le 28 novembre de cette même année, le président George W. Bush Sr. se félicitait de l'impulsion nouvelle qu'allait susciter l'existence d'un musée consacré aux premiers habitants du Nouveau Monde :

J'ai grand plaisir à signer aujourd'hui la loi S. 978 portant sur la création du National Museum of the American Indian. Cette loi ouvre la voie à une époque nouvelle qui permettra à la Nation de progresser vers une meilleure compréhension du patrimoine, de la culture et des valeurs des Amérindiens. ${ }^{3}$

Cette création ne s'est pas faite en un jour. Le transfert dans la capitale fédérale de la collection du Heye Museum de New York, connu sous le nom de "Indian Museum » (musée indien), a provoqué des débats et des affrontements tant parmi les politiques que parmi les anthropologues spécialistes des musées. Comme le rappelle Roland Force, son dernier directeur, l'histoire du Museum of the American Indian se résume en trois mots : «musées, argent et politique » 4 . Mais revenons en arrière.

\section{George Gustav Heye et la passion du collectionneur}

Né en 1874 et issu d'une famille de riches industriels, ingénieur de formation, George Heye fait l'acquisition en 1897 d'une première pièce - une chemise navajo 
en peau -, alors qu'il supervise le chantier de construction du chemin de fer le long de la frontière entre la Californie et l'Arizona. Désormais, la passion de la collecte ne le quitte plus, comme il le dit lui-même : " Naturellement, une fois que j'ai eu la chemise, je voulais aussi un hochet et des mocassins ${ }^{5}$. Au fil des années, il constitue la collection la plus importante du monde. Ce qui la caractérise, c'est non seulement la diversité des pièces ethnographiques et archéologiques qu'elle réunit, appartenant à plus d'un millier de groupes ethniques et couvrant une période de 10000 ans ${ }^{6}$, mais aussi la quantité exceptionnellement importante d'objets d'une même catégorie (mocassins, paniers, poteries, masques ou encore objets de la Côte nord-ouest). À l'époque, comme le souligne le directeur adjoint aux ressources culturelles, Bruce Bernstein, l'absence relative de concurrence- les grands musées américains et européens ayant alors achevé la constitution de leurs collections - donne à Heye la possibilité de «satisfaire son appétit vorace de collectionneur $~^{7}$. En soixante années, il rassemble en effet près d'un million d'objets.

Le Museum of the American Indian est fondé en 1916 ; il ouvre ses portes au public en 1922. De son vivant, Heye refuse obstinément que sa collection soit intégrée au fonds d'autres institutions. C'est ainsi qu'en 1914, il ignore les sollicitations de Franz Boas qui souhaitait qu'« il unisse [ses] efforts à ceux de l'American Museum of Natural History de New York ${ }^{8}$. Heye fait construire son musée à Audubon Terrace au carrefour de Broadway et de la $155^{\mathrm{e}}$ rue, dans un quartier alors réputé pour ses institutions artistiques et culturelles. Mais l'établissement devient vite trop exigu pour les quelque 400000 spécimens ${ }^{9}$. Il faut dès 1926 créer une annexe dans le Bronx pour les accueillir.

À sa mort, George Heye lègue la totalité de son fonds à la ville de New York qui ne semble pas disposée à soutenir les activités du musée. En raison de sa mauvaise localisation - le quartier de Washington Heights s'est progressivement dégradé -, d'un taux de fréquentation très bas, d'un manque de personnel et d'une muséographie pour le moins désuète, l'institution se trouve confrontée à une crise grave. De mauvaises conditions de stockage et de conservation des objets ne font qu'accroître les problèmes et, dans les années 1980, il est question de transférer le fonds Heye dans une autre ville ou une autre région.

\section{Une institution en déclin}

En 1974, un scandale qui concerne la vente d'objets à des collectionneurs privés est révélé par le New York Times. Cette pratique, acceptable du vivant de George Heye, quand la collection lui appartenait en propre, est alors jugée contestable car il s'agit désormais d'une collection publique. À cela s'ajoutent le manque de rigueur dans la gestion du fonds et la mauvaise tenue des inventaires du musée. Alors qu'il faut prendre des mesures pour le sauver, New York et ses édiles font la sourde oreille. Plusieurs villes proposent alors d'accueillir la 
Heye Foundation: Las Vegas, Indianapolis et Dallas, proposition qui a le soutien du politicien millionnaire Ross Perot mais qui suscite beaucoup d'opposition.

En 1982, l'annonce d'un possible transfert de la collection finit par émouvoir la municipalité et les New-Yorkais. Il est question notamment d'opérer une fusion avec le fonds de l'American Museum of Natural History, mais il faudrait aller contre les dernières volontés de George Heye. Se posent non seulement la question du statut et des conditions de conservation de la collection, mais encore celle de la place attribuée aux communautés amérindiennes, le conseil d'administration de l'American Museum ne leur proposant qu'un rôle de figurants. On se trouve devant une impasse et la controverse s'envenime. L'historien Alvyn Josephy et l'écrivain Vine Deloria Jr. donnent de la voix et déclarent publiquement que la collection fait partie du patrimoine amérindien ${ }^{10}$.

Le sort du musée reste incertain jusqu'en 1987, date à laquelle le sénateur démocrate de Hawaï, Daniel Inouye, présente un projet de monument dédié à la mémoire des Amérindiens. Ben Nighthorse Campbell, alors représentant démocrate du Colorado, vient joindre ses forces à celles d'Inouye et, ensemble, ils constituent le front de défense autochtone auprès du gouvernement pour qu'un véritable musée indien soit fondé. La grande victoire des Amérindiens consiste alors à obtenir, dans le cadre de la Smithsonian Institution, gardienne du patrimoine national, la création d'un musée au cœur de la capitale fédérale. Une des conditions de ce transfert était que le National Museum of Natural History s'engage à assurer le rapatriement des restes humains et des objets sacrés réclamés par leur communauté d'origine. Le Museum Act de 1989 est donc à l'origine du Native American Graves Protection and Repatriation Act ${ }^{11}$ (NAGPRA) de 1990.

Richard West, Cheyenne/Arapaho, est nommé directeur du nouvel établissement en 1990. Il est bientôt décidé que le bâtiment sera construit par l'architecte Douglas Cardinal (Blackfoot) et géré par un personnel à majorité autochtone. Le projet de construction du nouveau musée est assorti de dispositions relatives à la mise en place d'une antenne new-yorkaise installée dans l'ancienne maison des douanes (Custom House) au sud de Manhattan. Consacrée aux expositions temporaires, elle ouvre ses portes au public en 1994. Un centre de conservation et de recherche est établi à Suitland, dans le Maryland, qui accueille, à partir de 1999, l'ensemble de la collection Heye, à laquelle s'ajoutent les acquisitions d'œuvres contemporaines. La construction du musée proprement dit sur le Mall s'achève, en dépit de diffícultés liées à la démission de Douglas Cardinal, juste à temps pour l'inauguration prévue à l'automne 2004. On prévoit actuellement la constitution d'une quatrième unité - un musée hors les murs - pour favoriser les échanges entre les conservateurs du musée et les communautés indiennes. 


\section{Richard West et le concept de " musée vivant "}

Le National Museum of the American Indian a pour mission de présenter et d'interpréter, par des voix multiples, dont celle des autochtones eux-mêmes, tous les aspects des cultures indiennes comme un ensemble de phénomènes dynamiques et vivants.

(Richard West $1992^{12}$ )

Dès le début des années 1990, Richard West énonce sa vision d'un musée différent. Républicain, considéré comme conservateur, il adopte une position radicale dans la mise en œuvre de sa mission. Il insiste surtout sur le fait que les Indiens ne veulent plus être considérés comme des "vestiges historiques » ${ }^{13}$. Selon lui, il convient de «se débarrasser de la vision européo-centriste que le $\mathrm{XIX}^{\mathrm{e}}$ siècle avait de la culture indienne, une culture figée au point que son image n'a pas changé du tout jusqu'à aujourd'hui : celle du noble sauvage au teint cuivré qui s'élance à travers les Grandes Plaines dans un déploiement de plumes et de perles ». West tient à mettre fin à ce qu'il appelle "notre statut de "morts", "d'objets d'étude" et de "primitifs", pour le remplacer par un statut d'êtres humains à part entière, vivants et contemporains $" 14$.

L'histoire personnelle du directeur du NMAI n'est pas étrangère aux objectifs nouveaux qu'il défend. Né, comme il aime à le dire lui-même, "dans deux mondes à la fois », d'un père cheyenne, peintre et sculpteur, et d'une mère non indienne, pianiste de talent et professeur de musique, il choisit d'étudier le droit, car il lui semble que la profession d'avocat lui permettra de défendre les intérêts des communautés et de contribuer à transformer le regard porté sur celles-ci par la société majoritaire. Formé à l'université de Stanford, il fait partie de cette nouvelle élite indienne qui veut lutter à armes égales avec les politiques, les administrateurs et, dans le cadre du musée, avec la vieille garde des "white anthros » ${ }^{15}$, ces " grands-prêtres » qu'il considère comme les représentants d'une époque révolue. Il propose que les autochtones racontent leur propre histoire sans l'intrusion des experts et que les objets soient mis à la disposition des groupes à des fins cérémonielles ${ }^{16}$. Il défend le principe de la consultation des communautés et de la collaboration avec leurs représentants qui sont appelés à donner leur avis et à faire partager leur savoir. Par ailleurs, en tant que juriste, West est particulièrement attaché à l'application rigoureuse du NAGPRA. Il rappelle que nombre des ossements détenus par la Smithsonian Institution ont été volés sur les champs de bataille dans le cadre d'un projet anthropométrique entrepris au $\mathrm{XIX}^{\mathrm{e}}$ siècle : "L'armée a mutilé des cadavres cheyennes [parmi mes ancêtres], dit-il, pour ne prendre que les têtes en laissant les corps sur place » ${ }^{17}$.

Au fil des années Richard West s'est entouré d'une équipe qui partage ses convictions : personnalités indiennes du monde des arts et des lettres, telles que 
Vine Deloria Jr. et Scott Momaday, et, en particulier, dans le domaine des musées, Dave Warren, l'un des fondateurs de l'Institute of American Indian Arts (IAIA) de Santa Fe, George Horse Capture du musée Buffalo Bill de Cody (Wyoming) et George McMaster, du musée des Civilisations du Canada. West aftirme sa volonté de contribuer à créer un "musée vivant », orienté vers l'avenir : « Nous sommes toujours là, déclare-t-il, et nous apportons une contribution vitale à la culture et à l'art américains contemporains $"{ }^{18}$.

D'une époque à l'autre, on peut mesurer le chemin parcouru dans la conception muséologique. Un monde sépare George Heye, Euro-Américain animé par la passion des objets, et seulement des objets, et Richard West, Indien cheyenne, animé par la volonté de prendre une revanche sur l'histoire. Le fait que le second soit en quelque sorte l'héritier du premier illustre la prise de contrôle institutionnelle par les Indiens, qui se trouvent aujourd'hui aux postes de commande au lieu de demeurer des objets scientifiques.

Mais la question des connaissances et du savoir-faire muséographique demeure posée, alors que les équipes mises en place par West ont rompu avec la " vieille » école des anthropologues chevronnés de la Smithsonian, et notamment avec l'American Indian Program du National Museum of Natural History dirigé par JoAllyn Archambault, responsable de la recherche, des publications et des expositions. Celle-ci, elle-même indienne lakota (sioux), se démarque de la position extrême qui consiste à dire que les membres extérieurs à une société ne peuvent parler de cette société, ce qui inplique que les non-Indiens ne seraient pas habilités à parler des cultures indiennes. Toutefois, Archambault considère qu'il est légitime de vouloir restaurer la fierté des autochtones. Ce qui lui paraît regrettable, c'est que les commissaires du NMAI ont tendance à faire émerger une version simplifiée et sur-spiritualisée des Indiens et des cultures indiennes. Elle s'indigne de surcroît que les budgets accordés à son programme soient réduits à la portion congrue depuis la création du nouveau musée ${ }^{19}$.

Il est vrai que le nouvel établissement a coûté la somme de 219 millions de dollars, dont plus de 100 millions versés par le gouvernement fédéral, ce qui laisse la part du pauvre au National Museum of Natural History. Mais, sur cette somme totale, 95 millions proviennent également de fonds privés. Outre la participation de mécènes (Peggy et David Rockefeller), de fondations et d'entreprises, les tribus dotées de casinos prospères, notamment les Manshatucket Pequot, les Mohegan et les Oneida du Wisconsin, ont apporté une contribution importante (quelque 30 millions chacune sur dix ans).

\section{L'inauguration du musée : un grand pow wow inter-tribal}

C'est le 21 septembre 2004, veille de l'équinoxe, qu'a eu lieu l'inauguration du NMAI. Des communautés indiennes de l'ensemble du continent ont participé au cortège qui les a conduites du Château (Muséum d'histoire naturelle) jusqu'aux 
portes du musée. Dès l'aube, en costumes cérémoniels (Figure 1), les représentants des communautés indiennes de tous horizons se sont rassemblés sous les bannières tribales. Le peintre navajo Tony Abeyta avait esquissé dans les tonalités sable et turquoise du Nouveau-Mexique les grands panneaux figuratifs ou abstraits qui permettaient de s'orienter dans cette procession colorée. On a dénombré environ deux cent mille personnes. À la mi-journée les orateurs se sont succédé sur l'estrade centrale pour les discours offïciels, notamment les élus qui ont joué un rôle déterminant dans la création du musée, Daniel Inouye et Ben Nighthorse Campbell, puis des invités politiques de marque tels que le président péruvien Alejandro Toledo. En point d'orgue, Richard West a déclaré :

Nous vivons sur cette terre depuis des milliers d'années [...]. Nous devons et nous voulons faire partie intégrante de la culture américaine [...]. Le National Museum of the American Indian est un symbole de compréhension et de respect mutuels ainsi que de réconciliation culturelle. Bienvenue en terre indienne ! Bienvenue chez vous !

Une semaine de festival a suivi, au cours de laquelle des spectacles de danse et des concerts ont transformé le centre de la capitale en grand pow wow indien. Tous les éléments étaient ainsi réunis pour une auto-célébration festive et une nouvelle rencontre entre Indiens et non-Indiens.

\section{Une architecture insolite}

Le "New Kid on the Block», avec sa couleur ocre, ses flancs arrondis, sa silhouette à la fois massive et harmonieuse (Figure 2), se fait remarquer en conférant une note insolite à l'ensemble architectural des monuments officiels qui l'entourent. Certains observateurs font remarquer qu'il semble vivant et dégage une impression de force étonnante :

Le bâtiment s'élève au-dessus des ormes de l'esplanade comme une apparition monumentale. Ses murs aux larges courbes semblent dire: « Regardez-moi». Plus on le regarde, plus il donne à voir et à réfléchir. [...] Sur le plan architectural autant que philosophique, le nouveau bâtiment est empreint d'un magnétisme qui suscite un dialogue avec son environnement et cela avec une grâce étonnante. ${ }^{21}$

Orientée vers l'est, du côté du soleil levant, sa façade semble avancer comme la proue d'un navire, à la conquête d'une reconnaissance ofticielle longtemps attendue. Tout autour du bâtiment, l'aménagement paysager conçu par Donna House, ethno-botaniste navajo, est une reconstitution de l'environnement naturel des tribus indiennes (marécages, forêts, déserts) avec ses plantes originaires du continent américain telles que tabac, courge, maïs. De lourds blocs de pierres extraits d'une carrière au Québec, surnommés " Grands-Pères », symbolisent l'ancienneté de la présence indienne en Amérique. Autour du musée, des cérémonies sont organisées pour animer l'espace architectural. 


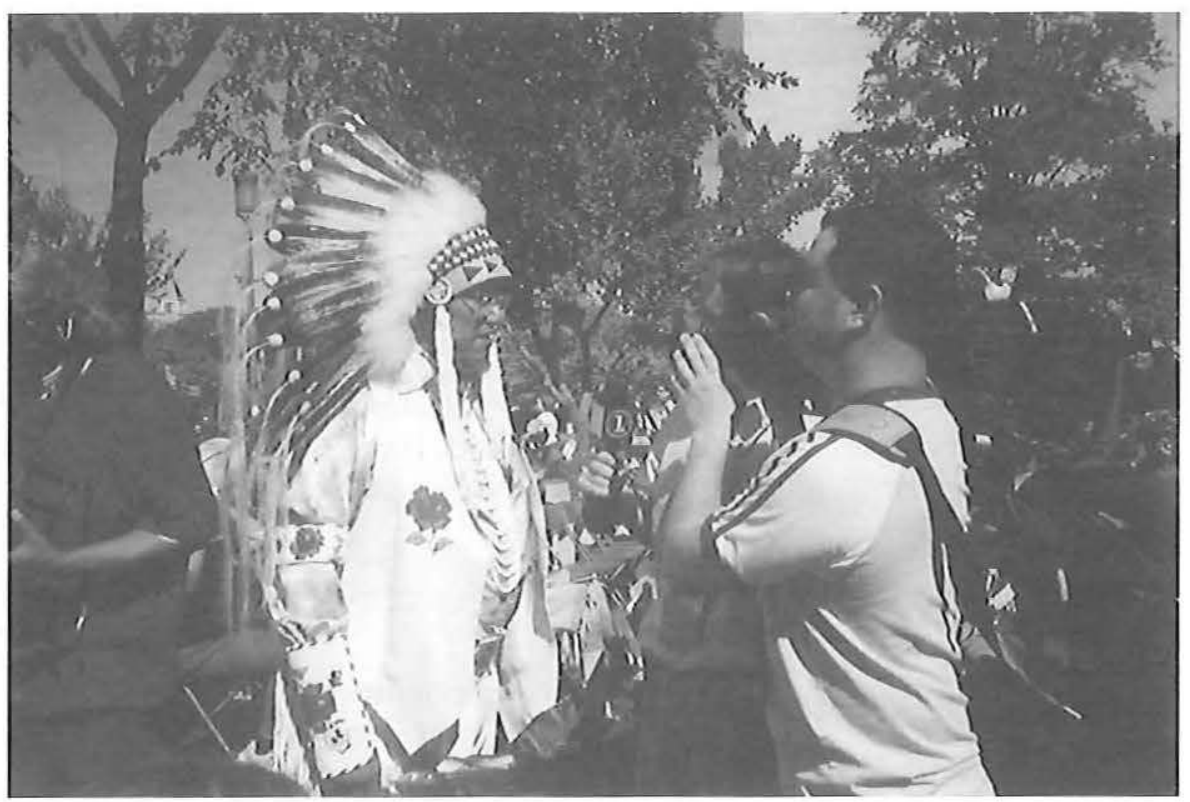

FIG. 1 - Pow wow sur le Mall (cliché Marie Mauzé)

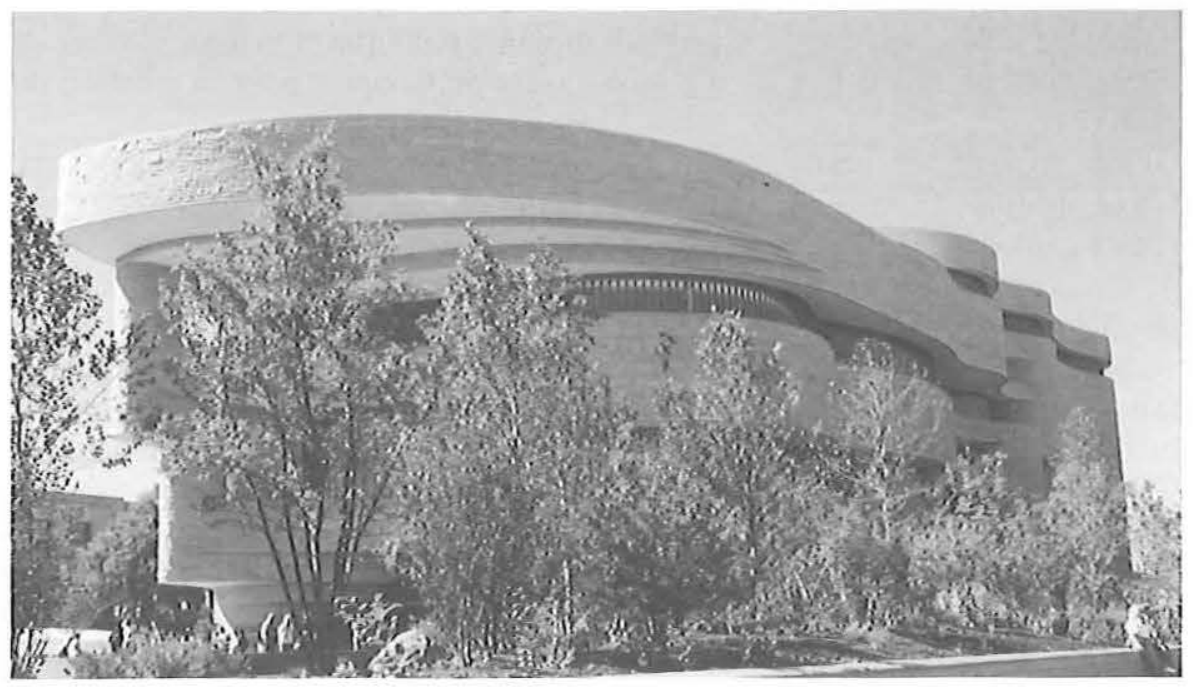

Fig. 2 - Le NMAI (cliché Nicolas Rostkowski) 
L'intérieur du bâtiment est spectaculaire mais son hall de réception, immense, donne une impression de grand vide. Appelé le Potomac (« là où l'on apporte les biens » en algonquin/powhatan), il accueille les animations culturelles. Pendant la semaine de l'inauguration, des artisans étaient à l'œuvre, fabriquant des canoës. Un escalier monumental conduit aux espaces d'exposition et le gigantesque dôme, d'où filtre la lumière naturelle, grâce à un jeu de huit prismes, projette sur les murs des couleurs d'arc-en-ciel. Un centre de recherche permet d'étudier les collections du musée et devrait être bientôt relié à d'autres établissements. Des films et des programmes audio-visuels sont proposés dans deux amphithéâtres. Deux (trop) vastes boutiques proposent livres et objets d'artisanat. Au rez-dechaussée le Mitsitam Cafe ("mangeons » en piscataway/delaware) permet un voyage gastronomique en terre indienne : steaks de bison, saumon sauvage de la Côte Nord-Ouest, maïs grillé et pain frit des Pueblos du Nouveau-Mexique sont au menu.

\section{Le parcours muséographique}

On distingue deux types d'expositions, les temporaires et les permanentes. Les deux présentations temporaires inaugurales sont consacrées à des artistes contemporains. La première, et la plus importante, propose une rétrospective des œuvres du sculpteur et dessinateur apache chiricahua Allan Hauser (1914-1994) et du peintre chippewa George Morrison (1919-2000). Ces deux grands artistes, en ouvrant de nouvelles voies à la création artistique amérindienne, ont su pénétrer le monde de l'art occidental. La seconde exposition temporaire rend hommage à Ben Nighthorse Campbell, homme politique et artisan joaillier de talent, dont on sait le rôle qu'il a joué auprès du Congrès pour la création du NMAI.

Les expositions permanentes sont structurées autour d'axes principaux. L'une, intitulée Windows on Collections. Many' Hands, Many Voices, s'attache à rendre compte de la richesse et de la diversité des œuvres dans le temps et l'espace, de la variété des formes et des matériaux en mettant en évidence le savoir-faire traditionnel des artistes amérindiens. Elle présente 3500 objets répartis en six vitrines, dont chacune traite d'un thème précis. Le visiteur pourra admirer, en raison de l'effet esthétisant qui s'en dégage, la vitrine regroupant environ un millier de pointes de flèches exposées de manière à figurer un envol d'oiseaux. Cette disposition n'est pas sans rappeler celle des objets disposés en panoplies qui a marqué la muséographie de la fin du $\mathrm{XIX}^{\mathrm{e}}$ et du début du $\mathrm{Xx}^{\mathrm{e}}$ siècle. Dans une vitrine, l'accumulation de poupées de l'ensemble du continent américain évoque les cabinets de curiosités ou encore les installations des musées d'histoire naturelle qui regroupaient les artefacts par catégorie et/ou par fonction. Le même parti-pris scénographique vaut pour la présentation d'ouvrages perlés aussi différents qu'un ornement de chanfrein du Nord-Dakota, un chapeau aléoute, des mocas- 
sins ou une pelote à épingles, ou encore pour celle d'une série pour le moins éclectique d'objets qui ont pour seul point commun de figurer des animaux (aigle, ours, etc.) dont on nous informe qu'ils ont joué un rôle important dans la vie des Amérindiens. Enfin, on ne manquera pas de s'arrêter devant la vitrine des médailles de paix (peace medals) placées au pied de portraits de grands chefs indiens parmi lesquels on reconnaît notamment Richard West, le directeur du musée, portant beau avec sa superbe coiffure en plumes. Le visiteur est invité à lire ceci :

Au moment de la révolution américaine, la tradition qui consistait à offrir des objets symboliques aux Amérindiens remontait à environ vingt années. Les officiers anglais, français et espagnols offraient aux chefs des médailles, des tomahawks, des drapeaux, des fusils pour maintenir la paix et gagner l'amitié des puissantes nations indiennes. Les États-Unis ont adopté et perpétué cette tradition jusque dans les années 1880.

Tout en s'appropriant une muséographie caractéristique des pratiques autrefois à l'honneur, les commissaires ont parié sur les nouvelles technologies (écran tactile, zoom électronique, vidéo) qui, certes, apportent une contribution ludique à l'observation des objets mais ne compensent évidemment pas le manque d'informations détaillées sur leur origine et leur fonction.

Trois autres expositions permanentes sont organisées chacune autour d'un thème : Our Universes (Nos univers), Our Peoples (Nos peuples), Our lives (Nos vies), chacune des trois sections donnant la parole à huit communautés situées entre l'Arctique et la Terre de Feu. À ce propos, on devine aisément que les critères de sélection des groupes ainsi représentés, sur des centaines qui auraient pu être choisis, tiennent à la situation géographique de quelques-uns (par exemple les Piscataway de la région de Washington), à la contribution financière qu'ils ont pu fournir (plusieurs sont propriétaires d'établissements de jeu) ${ }^{22}$, mais aussi à l'aura morale et intellectuelle d'éminentes personnalités telles que Scott Momaday (Kiowa) ou Dave Warren (Santa Clara Pueblo). On remarque que, parmi les vingt-quatre groupes retenus, un nombre relativement important est presque inconnu des spécialistes et a fortiori du grand public. Il convient également de noter que les communautés sélectionnées correspondent à des localisations clairement identifiées, de sorte que l'unité sociale de référence est bien la communauté, tout au plus la tribu, mais certainement pas un regroupement quelconque correspondant aux aires culturelles communément définies (Plaines, Woodlands, etc.).

Avec l'exposition Our Universes, sous-titrée « Notre savoir traditionnel donne forme à notre monde ", le visiteur est invité à découvrir la cosmologie amérindienne à travers le cycle d'une année solaire. Comme l'explique le commissaire Emil Her Many Horses (Sioux Oglala), elle marque de son empreinte la vie sociale et cérémonielle : 
[Nos ancêtres] nous ont appris à vivre en harmonie avec les animaux, les plantes, le monde des esprits et tous ceux qui nous entourent. Dans [l'exposition] Nos univers, vous rencontrerez des peuples autochtones qui continuent d'exprimer leur sagesse à travers les cérémonies, les fêtes, les langues, les arts, les religions et la vie quotidienne. Il est de notre devoir de transmettre ces enseignements aux futures générations. Car c'est une manière de garder nos traditions vivantes.

Les Ashinabe ont choisi une muséographie façon life-group mettant en scène des mannequins pour évoquer les rites de passage qui jalonnent les moments importants du cycle de vie des hommes et des femmes. Ils rappellent que les valeurs (ici appelées " enseignements ») qui sous-tendent leur vision du monde sont au nombre de sept (vérité, honnêteté, courage, respect, amour, sagesse, humilité). Les Hupa de Californie, quant à eux, attribuent à la white deerskin dance (danse du daim blanc) un rôle pivot dans leur système rituel. Le costume porté à cette occasion est mis en évidence sur un mannequin. Citons encore l'importance du calendrier dans la culture maya illustrée, en l'occurrence, par les Q'eqchi' de Coban.

Selon Duane Blue Spruce (Laguna Pueblo), Our Peoples a pour ambition de retracer " un pan d'histoire que les gens ne connaissent pas et ne voudraient probablement pas connaître » ${ }^{23}$. Il s'agit de "Faire parler nos histoires » ou, pour reprendre la formule de l'historien George Sioui, présenter une autohistoire. L'exposition met l'accent sur le choc qu'a constitué l'arrivée des Européens pour les premiers habitants des Amériques. Les faits de colonisation, les politiques gouvernementales et la christianisation sont abordés sous l'intitulé « La Tempête » par l'exposition de plusieurs centaines de fusils disposés dans une grande vitrine rectangulaire face à une autre contenant deux cents bibles ainsi que de très nombreux documents officiels liés notamment à la spoliation des terres. La " grande Histoire » que l'on trouve dans les livres n'a pas sa place au musée. L'accent est mis sur des fragments d'histoire locale de groupes particuliers. Des témoignages sont reproduits sur des panneaux, des écouteurs diffusent la mémoire des Anciens. La parole est donnée à des représentants de chaque communauté. Ainsi les Tapirapé du Brésil (Mato Grosso) déclarent : "Notre force vient de notre peuple et de notre terre ", et les Cherokee de Caroline du Nord : "Nous sommes toujours là », tandis que les Kiowa affirment leur attachement à « [leurs] chants et leurs cérémonies [qui les] aident à vivre ».

La troisième et dernière exposition permanente, Our Lives, est consacrée au mode de vie et à la question de l'identité au $\mathrm{xxI}^{\mathrm{e}}$ siècle. Est mise en valeur l'émergence d'une élite ancrée dans deux mondes à la fois, celui de la modernité et celui de la tradition. Ce thème, qui paraît banal, était certainement celui dont le traitement exigeait le plus de rigueur et d'à-propos, compte tenu de l'extrême complexité des problèmes contemporains. Ici, ce sont de grands portraits individuels de personnages saisis sur le vif qui sont censés illustrer les multiples facettes de la condition indienne. Le visiteur découvre le quotidien de diverses commu- 
nautés. On passe par exemple des Inuit d'Igloolik, avec la reconstitution d'une cuisine moderne, aux Indiens urbains de Chicago qui s'entourent chez eux de signes distinctifs de l'indianité, et aux Métis de la Rivière rouge du Manitoba dont la langue, le michif, est un mélange de français canadien, de saulteaux, de cree et d'ojibway.

La question de l'identité est posée avec insistance. Référence est faite au Red Power, mouvement indien de contestation des années 1960 et 1970, « quand après presque cinq cents ans d'occupation et de domination, les Indiens se sont enfin affirmés sur les plans culturel, spirituel, et politique ». Un slogan est mis en exergue : « La survivance est plus que la simple survie ». Il appartient à chacun d'en être l'artisan.

\section{Sacralisation des objets}

On peut s'interroger sur le fait que seulement 8000 pièces sur les 862000 que possède le musée soient actuellement exposées. La raison en est sans doute que pour les responsables du NMAI, les objets sont moins importants que la vie des communautés dont ils sont originaires. En vérité, les collections sont précieusement conservées au Cultural Ressources Centre (CRC) de Suitland dont le directeur adjoint, James Pepper Henry, fait remarquer qu'il est «l'âme du musée ». Même si ces pièces sont appelées à être présentées au public ultérieurement dans le cadre d'expositions temporaires, il demeure que la richesse n'apparaît pas dans la conception des expositions.

Bon nombre d'objets sont considérés comme culturellement sensibles et ne peuvent être montrés à certaines catégories de personnes en fonction de leur âge ou de leur sexe. C'est la raison pour laquelle le CRC n'est pas conçu comme un entrepôt mais plutôt comme une demeure. Le traitement qui est réservé aux objets, conforme aux méthodes les plus modernes de conservation, se fait aussi dans le respect des impératifs liés aux traditions. À plus forte raison quand il s'agit de pièces dotées d'une force spirituelle particulière et donc potentiellement dangereuses ou vulnérables. Certaines d'entre elles sont nourries, d'autres, comme les Kachinas, doivent pouvoir respirer à l'air libre, et d'autres encore sont bénies par fumigation rituelle. Les paquets-médecine, quant à eux, sont placés en hauteur, hors d'atteinte, car selon les conservateurs du CRC ils pourraient encore abriter des esprits qu'il ne faut pas déranger.

Dans le musée lui-même, la sélection repose sur l'importance spirituelle des pièces plutôt que sur leurs qualités esthétiques. Pour George Horse Capture (Gros Ventre) « si l'on croit dans le pouvoir des objets, on perçoit différemment l'importance d'une chemise de guerre [...] C'est une sorte de poème qui parle de la souffrance, des massacres, des maladies et de l'espoir : elle nous conte l'histoire d'un ancêtre ${ }^{24}$ ». Si l'on encourage les visiteurs à apprécier le pouvoir spirituel des objets, on utilise toutefois aussi cet argument pour les soustraire à leur curiosité. 
Le NMAI a été créé dans un contexte historique et politique particulier. La reconnaissance progressive des droits des peuples autochtones, au cours de ces trente dernières années, a eu des répercussions dans le domaine muséal et a fait évoluer la représentation des Premiers habitants du continent. Depuis les années 1980, les Amérindiens jouent un rôle croissant de collaboration et de conseil auprès des institutions muséographiques tant aux États-Unis qu'au Canada. Un certain nombre de centres culturels locaux ont vu le jour en territoire indien au cours de cette même période. Des institutions inter-tribales, telles que l'Institute of American Indian Arts (IAIA) de Santa Fe, ont formé toute une génération de jeunes artistes, à la fois déterminés à demeurer fidèles à leurs traditions et à s'intégrer au monde de l'art contemporain.

Au sein de la Smithsonian, on a assisté à la reconnaissance progressive des voix autochtones, mais l'institution s'est heurtée à la difficulté d'innover dans les anciennes structures néo-coloniales que sont les musées d'histoire naturelle. L'héritage presque encombrant de la collection Heye a permis de repenser la conception même d'un musée pour les Indiens et a fourni l'opportunité aux plus engagés d'entre eux d'en prendre les commandes. On assiste donc, avec l'inauguration du NMAI, à un événement majeur qui s'inscrit cependant dans un courant de pensée plus général ayant conduit à des changements de perspectives dans le monde muséal en Australie, en Nouvelle-Zélande et au Canada.

En remettant en question l'autorité du discours anthropologique, le NMAI entend rompre avec la tradition scientifique et la vision de l'histoire de la société dominante. Ce qui change, c'est le discours et le nouveau regard que les Amérindiens imposent au public. Mais on peut légitimement se demander si ce qui nous est donné à voir et à comprendre est à la hauteur des ambitions que le musée s'est fixées. En effet, sur le plan des installations muséographiques proprement dites, on ne constate aucune innovation majeure, si ce n'est, comme nous l'avons mentionné, que le musée fait appel aux ressources technologiques nouvelles. En outre, le visiteur, sollicité par la multitude des messages sonores, visuels et conceptuels, ne sait où porter son attention. Sans doute faut-il imputer à la " plurivocalité », clé de voûte de la conception d'ensemble des trois expositions permanentes, cette sensation de foisonnement d'informations incomplètes et répétitives. En définitive, si la parole est multiple, le discours qui la sous-tend serait unique. En voulant faire entendre de façon systématique les seules voix indiennes, on aboutit à un point de vue symétrique et inverse de celui des anthropologues. La question se pose de savoir si cette approche présentiste des cultures et des sociétés indiennes pourra se renouveler dans la longue durée et satisfaire les quatre millions de visiteurs attendus chaque année.

"Nous sommes les protecteurs de notre mère la Terre ». En martelant ce message d'inspiration écologique, le musée présente les cultures amérindiennes comme les gardiennes du présent et les inspiratrices de l'avenir. Cet avenir est défini en termes spirituels tant par la re-sacralisation des objets que par les 
cérémonies rituelles qui sont associées à leur mise en scène. Cette mise en exergue d'une écologie spirituelle aboutit à un processus d'essentialisation par lequel, au-delà des différences culturelles, émerge un homo spiritualus en parfaite harmonie avec son environnement naturel et le monde des esprits. Les vaincus de l'histoire deviennent un modèle vers lequel devrait tendre le reste de la société américaine.

Marie Mauzé, CNRS, Laboratoire d'anthropologie sociale,
Joëlle RostKowSKI, UNESCO/EHESS

\section{NOTES}

1. Selon l'expression de W. Richard West in National Museum of the American Indian, Smithsonian Institution Map and Guide, 2004, p. 7.

2. Jacqueline Trescott, " History's New Look », Washington Post, 13 septembre 2004.

3. Roland Force, 1999, The Heye and the Mighty, Politics and the Museum of the American Indian (Mechas Press, Honolulu, p. 445).

4. Force, op. cit., p. xvi.

5. Mason, 1958, p. 11, cité in Bruce Bernstein, 2004, «The National Museum of the American Indian Collections ", American Indian Art Magazine, Autum 2004, 29 (4), p. 52.

6. Mary Jane Lenz, National Geographic, septembre 2004.

7. Bernstein, 2004, art. cit., p. 53.

8. Force, 1999, p. 9. Voir correspondance Heye/Boas in ibid., pp. 469-470.

9. Force, op. cit., p. 9.

10. Force, op. cit., p. 136; Jerry Reynolds, " The Struggle to Save the Heye Collection ", Indian Country Today, 22 septembre 2004, p. B5.

11. Cette loi fait obligation aux musées subventionnés par le gouvernement fédéral de restituer sous certaines conditions aux communautés qui en font la demande les ossements et les objets sacrés. Suzan Harjo, alors directrice du National Congress of the American Indian et membre de l'American Indian Heye Foundation, joue un rôle clé dans l'adoption d'une résolution visant au rapatriement des restes humains en possession de la Smithsonian Institution. Voir " Inouye's Remarks on S. 978 », Indian Country Today, 22 septembre 2004, p. A8.

12. Richard West, 1992, "Entre deux mondes. Vers une nouvelle conception des musées d'art indien ", in Sylvie Devers et Joëlle Rostkowski, éds, Destins croisés. Cinq siècles de rencontres avec les Amérindiens (Albin Michel/UNESCO, Paris, p. 328).

13. Thomas Hayden, « By the People ", Smithsonian, septembre 2004, p. 52.

14. In Sylvie Devers et Joëlle Rostkowski, éds, op. cit., p. 328.

15. Jerry Reynolds, art. cit., "The Struggle to Save the Heye Collection », p. B1.

16. Edward Rothstein, "A Museum that Speaks with an American Indian Voice ", New York Times, 21 septembre 2004, p. B5.

17. In Sylvie Devers et Joëlle Rostkowski, éds, op. cit., p. 324.

18. Barbara Wells, "NMAI Revolves Around Themes: Native Universes, Peoples, Lives ", The Torch, septembre 2004, p. S1.

19. Bob Thompson, « Spirit Lodge », Washington Post, 2 août 2004, p. C2.

20. Note de terrain. Allocution de R. West le 21 septembre 2004.

21. Benjamin Forgey, « Natural Wonder », Washington Post, 17 septembre 2004, p. Cl.

22. À titre d'exemple, les Cherokee de l'Est ont participé à hauteur de 250000 dollars. Un des objets représentés dans l'exposition qui leur est consacrée est un document du début du XIx ${ }^{e}$ siècle qui 
établit la reconnaissance fédérale de leur tribu, USA Today, 22 septembre 2004, p. A16, in « Day of Celebration Ushers in Museum " par Raju Chebium et Faith Bremner. On peut citer aussi les Séminole, les Yakima ou la bande Campo de Californie.

23. Thomas Hayden, « By the People », Simithsonian, septembre 2004, p. 55.

24. Thomas Hayden, ibid., p. Cl.

\section{L'IDENTITÉ INDIENNE À L'ÉPREUVE DE LA MODERNITÉ}

Avec l'élection du républicain George W. Bush à la présidence des États-Unis en novembre 2000, et celle d'un Congrès majoritairement républicain, le budget fédéral en matière d'affaires indiennes a sensiblement diminué par rapport à celui des années Clinton. Pourtant, dans le discours du président actuel, il est question de la relation privilégiée qu'il dit vouloir maintenir avec les Amérindiens, très courtisés en cette période électorale 2004.

Pour la première fois dans l'histoire des États-Unis, le vote autochtone pourrait bien faire basculer les résultats dans tel ou tel État membre - dont le Nouveau-Mexique $(9,5 \%$ de la population), l'Arizona ( $5 \%$ ) ou le Nevada $(1,3 \%)$ - et, donc, in fine, dans l'élection même du président. Ce poids électoral des Amérindiens découle d'une reconstruction démographique, économique et culturelle enclenchée dans les années 1960 et qui, depuis, n’a fait que se renforcer, particulièrement avec la prolifération des casinos indiens au début des années 1980. Il existe à ce jour plus de 350 casinos indiens, répartis dans 200 réserves et 30 États membres ${ }^{1}$. Mais cette émergence politique sur la scène nationale s'accompagne de nouveaux problèmes - administratifs, juridiques, politiques et culturels -, eux-mêmes aggravés par les attentats du 11 septembre 2001 et l'entrée en guerre des États-Unis contre l'Irak. La mondialisation n'épargne pas les Amérindiens.

La première GI américaine morte au front était hopi. Il s'agit de Lorri Piestewa, qui se trouvait dans la même embuscade que Jessica Lynch, devenue célèbre grâce au montage mensonger, mais lucratif, de son « évasion ». Fille d'un ancien combattant du Vietnam et petite-fille d'un ancien combattant de la Seconde Guerre mondiale, Lorri avait 23 ans. Mais il ne fut jamais mention d'elle dans la presse grand public. Pourtant, la contribution des Amérindiens à cette guerre (comme à toutes les guerres américaines, particulièrement la Seconde Guerre mondiale et la guerre du Vietnam) est importante. Ainsi, envoyés récemment au combat, 600 membres de la nation cherokee d'Oklahoma ont décidé 